Revue scientifique sur la conception et l'aménagement de l'espace

19 | 2018

L'imagerie du paysage

\title{
Présentation de la rubrique Matières premières
}

Jean-Luc Brisson et Sabine Ehrmann

\section{OpenEdition}

\section{Journals}

Édition électronique

URL : http://journals.openedition.org/paysage/293

DOI : $10.4000 /$ paysage.293

ISSN : 1969-6124

\section{Éditeur :}

École nationale supérieure du paysage de Versailles-Marseille, Institut national des sciences appliquées Centre Val de Loire - École de la nature et du paysage, École nationale supérieure d'architecture et de paysage de Bordeaux, École nationale supérieure d'architecture et de paysage de Lille, Agrocampus Angers

\section{Référence électronique}

Jean-Luc Brisson et Sabine Ehrmann, «Présentation de la rubrique Matières premières », Projets de paysage [En ligne], 19 | 2018, mis en ligne le 23 décembre 2018, consulté le 25 septembre 2020. URL http://journals.openedition.org/paysage/293 ; DOI : https://doi.org/10.4000/paysage.293

Ce document a été généré automatiquement le 25 septembre 2020

Projets de paysage 


\title{
Présentation de la rubrique Matières premières
}

\author{
Jean-Luc Brisson et Sabine Ehrmann
}

1 Si les dossiers thématiques rassemblent les contributions à caractère scientifique, la rubrique "Matières premières" accueille des témoignages ou des recensions d'expériences, tous types de documentations émanant d'un-e professionnel-le à propos de son propre travail. Il s'agit ici de contributions dont la distance critique n'est pas assurée mais qui sont mises à la disposition de la communauté des chercheurs-euses pour des développements ultérieurs. La rubrique « Matières premières » de ce numéro 19 était, compte tenu du sujet de son dossier thématique, particulièrement ouverte à des contributions visuelles, et se proposait d'accueillir avec intérêt des «imagiers » inédits. Elle comporte deux contributions : celle de Sophie Goupille, qui nous livre son expérience personnelle de la frontière franco-belge et ses réflexions quant aux moyens de représenter et de partager cette expérience, et celle de Florence Bœuf et Frédéric Robert qui partagent ici les « herbiers de paysage » qu'ils confectionnent dans le cadre de leur activité de paysagistes. Ces deux contributions ouvrent des perspectives de recherche diamétralement opposées. Présentées côte à côte, elles bornent le spectre des usages et des problématiques qui lient l'imagerie au projet de paysage : d'un côté la perspective d'une objectivation des données de l'expérience, de l'autre l'inspiration factuelle et plastique pour la détermination du projet.

2 L'ensemble des images livrées par Sophie Goupille relève d'une procédure de représentation bien connue des géographes quantitativistes. Il s'agit d'un protocole de prises de vue photographiques rigoureux - « Tous les cent-cinquante pas, je décide de réaliser deux clichés perpendiculairement à la ligne, un à gauche, un à droite » - qui tente d'échapper à la subjectivité des points de vue en les remplaçant par une démarche arbitraire et systématique censée atteindre à l'objectivité de la représentation, ou du moins de garantir sa neutralité. Ce protocole de prise de vue réactualise ainsi paradoxalement dans le champ scientifique ou pseudo-scientifique le mythe de l'homme-caméra de Dziga Vertov, homme débarrassé par la technique de son propre regard. Aussi on pourra s'étonner de voir ici ce protocole qui tend à la 
désincarnation de l'observateur couplé à un récit mené à la première personne et employé dans la perspective inédite d'atteindre à la représentation d'une "matière première perceptive ». On voit alors, exemplairement, se constituer un bricolage méthodologique et figural qui tente de conjuguer données factuelles et données de l'expérience ; bricolage qui travaille en profondeur n'importe quel projet et n'importe quelle recherche concernant le paysage et qui constitue l'une des problématiques les plus stimulantes et les plus épineuses de la recherche visuelle en paysage.

3 Avec les imagiers livrés par Florence Bœuf et Frédéric Robert, nous nous situons à l'autre bout du spectre des relations entre imagerie et paysage. Ces petits cahiers $20 \mathrm{x}$ $20 \mathrm{~cm}$ ne tendent à aucune objectivité de la représentation. Tout au contraire ils se présentent comme le lieu de dépôt et le moyen de partage de la sensibilité et de l'imaginaire des projeteurs-euses. Ils n'en sont pas moins le chaudron d'un autre "bidouillage" où se fabriquent et se confondent des usages et des fonctions très différents de l'image. Tout à la fois catalogues de formes et de matières aptes à nourrir l'imagination et l'inspiration des projeteurs-euses, carnets de voyage véhiculant leurs visions esthétiques et poétiques, manuels pédagogiques à destination des partenaires capables d'attirer leur « attention sur les questions environnementales » et à " accepter l'incontrôlable, la diversité du monde végétal ", ces livrets font office de couteau suisse du projet. Ils interviennent autant comme outil de conception, de recherche que de communication et illustrent bien la difficulté à distinguer, dans l'exercice du projet de paysage, les motifs de l'inspiration formelle, de la rhétorique et de la publicité. Cette pratique des images, courante dans le milieu du design et de la mode, qui confond recherche formelle et recherche conceptuelle, bien qu'issue de manipulations essentiellement plastiques et graphiques, ne poursuit pas qu'une performativité formelle. Elle opère comme un agent d'influence qui tend in fine à assigner une répartition des rôles et à délimiter un pré-carré professionnel. Il s'y expose le paradoxe d'une appropriation du "sensible» et de l'«émotionnel », mise au service d'une stratégie de communication et de conviction, dont on pourra chercher à repérer, dans d'autres pratiques d'agence, les régularités et les modulations.

\section{AUTEURS}

\section{JEAN-LUC BRISSON}

evaporationmotrice[at]rocketmail[dot]com

\section{SABINE EHRMANN}

Sabine Ehrmann est artiste, docteure en esthétique, enseignante à l'École nationale supérieure d'architecture et de paysage de Lille (ENSAPL), chercheuse du Laboratoire Conception Territoire Histoire Matérialité (LACTH)

s-ehrmann[at]lille.archi[dot]fr 